\title{
LIGHT POLLUTION MODELING
}

\author{
R. H. GARSTANG \\ Joint Institute for Laboratory Astrophysics \\ University of Colorado and National Bureau of Standards \\ and Department of Astrophysical, Planetary and Atmospheric Sciences \\ and Department of Physics, University of Colorado \\ Boulder, CO 80309-0440
}

\section{INTRODUCTION}

The growth of urban development and its accompanying outdoor lighting has made the search for new observatory sites increasingly difficult. A method of predicting the brightness of the night sky produced by a city of known population and distance is useful in making studies of prospective new observatory sites, as well as in studying the likely future deterioration of existing sites. Other sources of light pollution can be investigated using the same model. In most cases, several cities are responsible for the light pollution at a given site, and the predicted night sky brightness is the sum of the contributions of all the cities. In this paper, we shall review the surprisingly little work which has been done on predicting night sky brightnesses from model calculations.

\section{OBSERVATIONAL DATA}

There is rather little observational data available for use in constructing and testing models for night sky brightness calculations. There are measures of the night sky brightness at major observatories, and there are some at prospective observatory sites, but published measurements are not extensive and often not very systematic. They will in due course prove of major importance when studying long term variations in the brightness such as may, for example, be caused by the sunspot cycle or by a secular increase in man-made pollution. Some of these measurements are also valuable for calibrating models. Measurements by Walker $(1970,1973)$ at Lick Observatory, Mount Palomar Observatory and Junipero Serra Peak (and at four other sites of no concern for the present review) are of importance for the latter reason. There are relatively few published measurements of the variation of the brightness caused by a city as a function of the distance of the observer from the city. Bertiau, de Graeve and Treanor (1973) gave measurements for Rome and for two other small towns in Italy, and Berry (1976) studied the area around Hamilton, Ontario, as well as other sites in Ontario. Walker (1977) made measurements at a series of distances from San Jose, Salinas and King City in California. Measurements of 
this kind provide a much stronger check on the accuracy of a model, and may enable the determination of the values of some parameters in a model which would not be readily determinable otherwise. We shall return to these studies after we have described the models which have been used.

\section{MODELS}

Walker: Walker (1970) measured the distance from San Jose at which the artificial brightening of the night sky at the zenith was $0.1 \mathrm{mag} / \mathrm{sec}^{2}$. He combined this measurement with measurements at Mount Palomar and with the assumption that the artificial brightening due to a city is proportional to its population. He obtained an approximate relationship giving the distance D from a city at which the zenith brightening caused by the city of known population $P$ is $0.1 \mathrm{mag} / \mathrm{sec}^{2}$. Walker (1973) revised this population-distance relationship using better population estimates. Although Walker did not mention it in these early papers, it is easy to show that his curve may be expressed to a remarkable accuracy as $P \propto D^{2.5}$. This is the simplest form of the population-distance relation. In later work, this relation was sometimes given for a brightness increase of $0.2 \mathrm{mag} / \mathrm{sec}^{2}$ at a zenith distance of $45^{\circ}$ towards a city. The I.A.U. has suggested (Smith 1979) as a standard criterion for a dark site that one should require the contribution of man-made light not to exceed 10 per cent of the natural background at any wavelength between $3000 \mathrm{~A}$ and $10000 \mathrm{~A}$ (except for the sodium $D$ lines for which a different criterion is suggested) at a zenith distance of $45^{\circ}$ towards a city. The criterion is approximately a brightness of 0.1 $\mathrm{mag} / \mathrm{sec}^{2}$.

The night sky brightness I caused by a polluting city is proportional to the number of lumens $\mathrm{L}$ emitted by the city, so that $\mathrm{I} \propto \mathrm{L}$. Walker (1977) obtained the total light emission L from several California cities and showed that the total light emission of a city is approximately proportional to the population of the city, so that $L \propto P$. There were a few exceptions to this statement, including some residential areas with very low light emission, and some industrial areas with higher emission. Some indications were obtained that a law $\mathrm{L} \propto \mathrm{P}^{0.8}$ might be a better approximation, but the uncertainties were such that it probably suffices to take $\mathrm{L} \propto \mathrm{P}$. This may be called the luminosity-population relation. Then we have $\mathrm{I} \propto \mathrm{P}$. Salinas is a large enough city that brightness measurements can be made to a reasonably large distance. Walker used his measures of the sky brightness as a function of distance from Salinas to show that the brightness follows the relation I $\propto \mathrm{D}^{-2.5}$ to remarkably good accuracy. This relation is the simplest form of the brightness-distance relation. Walker used his new measurements to improve his determination of the population-distance relation, and essentially confirmed the relation $P \propto D^{2.5}$ for producing a given sky brightness. These relationships may be combined into the single relation

$$
\mathrm{I}=\mathrm{CPD}^{-2.5}
$$

where $C$ is a coefficient which does not depend on $P$ and $D$ (but which depends on factors such as the light emission per head of the population and the reflectivity of the ground). We may regard this relation as a summary of the general results of Walker, and it can be regarded as the simplest model of light 
pollution. The explicit form of equation (1) was emphasized by Smith (1979).

Treanor: Treanor (1973) derived a formula for scattering by aerosols. He considered a simple model consisting of a homogeneous atmosphere in which the relevant vertical heights are small in relation to the horizontal distance between city and observatory. Cities were treated as point sources. He assumed that the light reaching a volume element of the atmosphere more or less in the zenith of the observer consists of two parts. The first is a direct beam from the city to the atmospheric element, the beam being subject to the inverse square law of distance and also to atmospheric extinction. The second part consists of light scattered by aerosols between the city and the atmospheric element. This scattering is limited to a cone of small angle about the forward direction of the beam. The beam intensity is subject to the inverse square law of distance and to atmospheric extinction. The scattering can take place anywhere along a path between the city and the atmospheric element. This leads to an integration along the path which introduces an additional power of the distance, so that the intensity reaching the atmospheric element is proportional to the inverse first power of the distance. The total intensity at the volume element is the sum of the two parts. A portion of the total is scattered down to the observer largely by Rayleigh scattering.

When the formulae for the contributions of single (Rayleigh) and double (Rayleigh and aerosol) scattering are combined into a single formula the resulting expression is written

$$
I=\operatorname{fP}\left(U D^{-2}+V D^{-1}\right) \exp (-k D)
$$

In this formula $I$ is the zenith night sky brightness at a distance $D$ from a city of population $P, k$ is an extinction coefficient, and $U$ and $V$ are constants. $f$ is a constant which Treanor introduced to take into account the light emission per head of the population, which depends on the state of economic development of a city. The term $U / D^{2}$ arises from Rayleigh scattering and the term V/D arises from Rayleigh and aerosol scattering.

Berry: Berry (1976) used a modification of the model of Treanor. Guided by his observations (discussed below), he used the square root of the population instead of the population. He also replaced the distance $\mathrm{D}$ of the city with $\left(\mathrm{D}^{2}+\right.$ $\left.\mathrm{h}^{2}\right)^{1 / 2}$ in equation (2), which is equivalent to assuming that the scattering all occurs at a height $h$ above the ground. His model also assumed that cities are point sources, that all cities are equally bright on a per capita basis (i.e. $f=1$ in equation (2)), and that all light is emitted by cities with negligible contribution from rural sources. Berry's model increases the importance of satellite cities relative to a main polluting city in comparison with models which assume that the light emission is proportional to the population.

Thomas, Modali and Roosen: In a brief abstract Thomas, Modali and Roosen (1973) reported calculations on night sky brightnesses using a Monte Carlo method, including a full treatment of polarization, a layered model atmosphere, and user-input density and size distribution of aerosols. The principal conclusion was that the combination of single and multiple scattering led to brightnesses about the same as those for a pure Rayleigh scattering 
atmosphere over a wide range of city-observatory separations. The present writer has been unable to find later publications on the subject by these authors.

Yocke, Hogo and Henderson: These authors (1986) applied an approximate treatment of radiative transfer to a study of the effect of a proposed nuclear waste depository on the night sky brightness as seen from sites in Canyonlands National Park. Their method has apparently not been applied to any observatories and sites.

Garstang: Garstang $(1986,1988 \mathrm{~b})$ set out to study the light pollution at particular sites, rather than making large scale survey calculations. He constructed a model based on much more detailed geometrical and physical considerations. In the first place, he felt that the assumption of a homogeneous atmosphere was rather inaccurate. For observatories at good sites polluted by distant cities, much of the light scattering takes place in the high atmosphere, often at several molecular scale heights above the ground. He postulated an atmosphere in which the densities of molecules and aerosols decreased exponentially with height, but with different scale heights for molecules and aerosols.

Rayleigh scattering was assumed for the molecular component of the atmosphere. Aerosols were represented by one kind of aerosol, with a conventional angular scattering function which showed the strong forward scattering of aerosols. A parameter $\mathrm{K}$ was introduced to represent the number of aerosols in the atmosphere, and hence the clarity of the atmosphere. The definition adopted was

$$
\mathrm{N}_{\mathrm{a}} \mathrm{r}_{\mathrm{a}}=11.778 \mathrm{KN}_{\mathrm{m}} \mathrm{r}_{\mathrm{R}} \exp (-\mathrm{cH})
$$

where $\mathrm{N}_{m}$ is the density of atmospheric molecules at sea level, $r_{R}$ is the Rayleigh scattering cross section of air at the wavelength of light of interest, $c$ is the reciprocal scale height of the molecular atmosphere, $\mathrm{H}$ is the altitude of the ground above sea level, $\mathrm{N}$ is the density of aerosol particles at ground level, and $r$ is the integrated scattering cross section of a single aerosol particle. The numerical factor was introduced to make $K=1$ for a standard sea level atmospheric model. Provision was made in the geometry for the observatory to be on top of a mountain at a specified height $A$ above the ground altitude $H$. To increase the accuracy of the calculations without the necessity of going to a full radiative transfer calculation, Garstang (1984) refined Treanor's formula. The possibility of using an atmosphere with an exponentially decreasing density had been considered by Bertiau et al. but they did not adopt it because of computational limitations. In addition, there was no reason to limit the calculations to the observer's zenith. Garstang showed that the assumption of an exponential atmosphere allowed the integration along the beam to be done analytically, and that equation (2) above should be replaced by

where

$$
\mathrm{I}=\mathrm{fPUD}^{-2}(\mathrm{DS})(\mathrm{EF})
$$

$$
(D S)=1+\frac{N_{a}{ }_{a}\{1-\exp (-a D \cos z)\}}{a \cos z}
$$


Here $\mathrm{f}, \mathrm{P}, \mathrm{D}, \mathrm{N}$ and $\mathrm{r}$ have the same meanings as before, $\mathrm{a}$ is the reciprocal scale height of the aerosols in the atmosphere, $\mathrm{z}$ is the zenith distance of the light beam from the city and $U$ is a constant. (The ratio $V / U$ of the constants in Treanor's formulation has in effect been evaluated in terms of the aerosol density and scattering cross section.) The constant $U$ can be interpreted as including the light emission per head of the population in the direction of the atmospheric element being considered. If desired the constant $f$ can be absorbed in $U$, and $U$ allowed the possibility of varying from city to city. (DS) is the factor which arises from the introduction of aerosol scattering between the city and the element of atmosphere. Garstang called it the double scattering correction, and introduced it whenever appropriate into his calculations. (EF) is the extinction factor, which replaces the factor $\exp (-\mathrm{kD})$ in equation (2). (EF) is calculated by integrating the extinction along the path of the light using the variation of the extinction with altitude above the ground given by the model atmosphere. For the path from a city to a volume element of atmosphere at a height $h$ above the ground we find that

$$
(E F)=\exp \left(-N_{m} r_{R} \exp (-c H) p \sec z\right)
$$

with

$$
\mathrm{p}=\mathrm{c}^{-1}\{1-\exp (-\mathrm{ch})\}+11.778 \mathrm{Ka}^{-1}\{1-\exp (-\mathrm{ah})\}
$$

A more general expression given by Garstang can be used for other situations. The angular distribution of the light emission from city lights was represented by a formula which contained as a parameter the fraction of the city light which is radiated above the horizontal, and so directly into the sky without an intervening ground reflection. This allowed the effect of improving the shielding of city lights to be investigated. The downgoing light was assumed to be reflected from the ground with a Lambert distribution. The reflectivity of the ground was included as a parameter: this allowed the effect of snow cover to be studied. The unshielded component of the city light which escapes upward without reflection from the ground was assumed to have a distribution with zero intensity towards the zenith and a distribution for larger zenith distances proportional to the fourth power of the zenith distance. (This is a completely arbitrary assumption, justified only by its reasonableness: if you look out from a plane as you land at night you get the impression that the intensity of the escaping light increases rapidly as poorly shielded street lights are seen more and more nearly horizontally.)

An approximate model for the night sky background was incorporated into the calculations, taking into account both faint stars and the airglow, and the intensities of these could be varied if desired. The night sky background was normalized to agree with the value at Junipero Serra Peak observed by Walker (1973). An improved model of the natural background was subsequently developed which takes into account light transmitted along the cone of vision and light scattered into the cone of vision by Rayleigh and aerosol scattering from the whole sky.

While a flat earth approximation is adequate for many calculations, curved earth geometry is needed for pollution by large distant cities (Garstang, 1988b). 
Curved earth geometry automatically allows for cases where the polluting city is beyond the optical horizon but city light is scattered to the observatory from the high atmosphere. Curved earth geometry also must be included in the double scattering and extinction calculations for large zenith distances of observation. Detailed formulae may be found in Garstang's paper.

In his earlier work Garstang (1986), had the city light emission expressed in lumens per head and obtained the sky brightness in nanolamberts and in magnitudes per square second of arc. In his later work, Garstang (1988b) modified his computer programs so that the light emission could be expressed in photons per second and the sky brightness in photons per second per square centimeter per steradian as well as in magnitudes per square second. Modifications were also made to give results for the B photometric band as well as for the $\mathrm{V}$ band and for the eye which alone had been used in the earlier work.

\section{APPLICATIONS OF MODELS TO THE ANALYSIS OF DATA}

Walker (1970) used his population-distance relationship to make a preliminary survey to delineate those areas of California which are too close to a large city to be considered for future observing sites. Walker (1973) used his revised population-distance relationship to improve his survey of California and he also made a survey of Arizona. He showed that substantial areas of both states are affected by light pollution to an extent that restricts future choices of observing sites in those states.

Bertiau et al. (1973) applied equation (1) to a study of the light pollution over most of Italy. They made a series of measurements of night sky brightness at various distances up to $40 \mathrm{~km}$ from Rome (population 2,600,000), at distances up to $21 \mathrm{~km}$ from L'Aquila (population 61,000) and at distances up to $5 \mathrm{~km}$ from Teramo (population 48,000). Teramo was chosen as an example of a medium size city which was less economically developed than L'Aquila. The measurements were made in the B wavelength band. Bertiau et al. showed that the observations for Rome and for L'Aquila satisfy the formula above with the same values of the constants $\mathrm{U}=13.5, \mathrm{~V}=1.8, \mathrm{k}=0.026$ and $\mathrm{f}=1$. Furthermore the observations for Teramo fitted the same curve with the same values of $U, V$ and $k$ if the value $f=0.77$ was chosen. The value of $k$ found is in reasonable agreement with $\mathrm{B}$ band extinction coefficients. Thus with an appropriate choice of $f$, unity for large towns and somewhat less than unity for underdeveloped towns, a formula had been obtained of universal validity.

Bertiau et al. used their formula and constants to calculate the brightness due to 1301 Italian towns at the centers of a mesh of squares covering the whole of Italy, each square having a $15 \mathrm{~km}$ side. The constant $\mathrm{f}$ was determined from the statistics of telephone ownership, a convenient measure of economic development. Over most of Italy, the sky brightness is unacceptably high for a major observatory. The only dark areas are the island of Monte Cristo, a large part of Sardinia, and a region in southern Italy near latitude $40^{\circ}$.

Berry (1976) organized a series of observations by members of the Royal Astronomical Society of Canada in Southern Ontario, using specially constructed portable photometers which could be carried by automobile. He pointed out the interesting fact that the sky near the horizon is brighter in directions both towards and away from a light polluting city. Horizon to horizon scans were 
made in downtown Toronto and at several other sites in Ontario. For all significantly polluted sites, Berry found the same shape of the horizon to horizon sky brightness curves as functions of zenith distance. Zenith measurements were made at the centers of a dozen cities of various sizes. The city center brightness was shown to be proportional to the square root of the population. This conclusion probably results at least in part from the extinction of that portion of the scattered light which originated in the outer parts of the larger cities, but we believe that geometrical factors are also important. Measurements were also made along a number of highways near Hamilton and at other sites. From an analysis of the data, Berry found that $\mathrm{U}=2.59, \mathrm{~V}=0.08, \mathrm{k}=0.026 \mathrm{~km}^{-1}$ and $\mathrm{h}=$ $2.4 \mathrm{~km}$.

Berry applied his model to Southern Ontario. He included a total of 325 cities, including Ontario cities with populations exceeding 1000, some cities in Quebec and some in nearby states of the U.S.A. Ontario was divided into 6000 blocks, each $8 \mathrm{~km}$ square, and the light pollution was calculated for all the blocks. The conclusions were clear. There are essentially no dark sites in the populated part of Ontario. An increase in the sky brightness due to man-made sources of $0.1 \mathrm{mag} / \mathrm{sec}^{2}$ or less occurs only in the Algonquin Park and northwestern Georgian Bay areas. Pike (1976) applied Berry's model to the prediction of future night sky brightness, estimated both the population growth rate for each city separately and the future growth of the light output per head of the population. The calculations and predictions extended to the year 2000 . The work again clearly showed that there will soon be no sites of adequate quality for the more demanding kinds of astronomical observations.

Garstang: Garstang (1986) applied his model to many situations. He studied the brightness-zenith distance relation for observations made at some distance from a city, and his calculations reproduced the observation by Berry (1976) that the sky brightness increases with zenith distance in directions away from the city as well as towards it. Calculations for the center of Toronto gave results in good agreement with Berry's observations. Garstang studied the brightness-distance relation. He showed that the brightness of the sky seen near the center of a city increases as the aerosol content of the atmosphere increases, but that outside the city increasing the aerosol content leads to lower man-made sky brightnesses. Calculations of the night sky brightness at various distances from Salinas gave a brightness-distance relation in substantial agreement with the observations of Walker (1977). The relationship was not quite a power law, but nevertheless was in reasonable agreement with the $D^{-2.5}$ law of Walker. Indications are that for large distances from a city the brightness falls off more rapidly than this law would indicate. Calculations were also performed on the population-distance relation, with results in good agreement with Walker (1977). The later work of Garstang (1988b) showed that the inclusion of curved earth geometry made only slight differences to the previous results on the brightnessdistance and population-distance relations. Curved earth geometry has no effect on vertical extinction calculations and little effect on ground level horizontal visibility calculations, but it does have a significant effect on horizontal visibility calculations from mountain observatories.

Garstang (1986) made a series of calculations of the brightness of the sky as seen from the centers of cities of various populations to try to clear up the difference between the assumption of luminosity proportional to the population 
(all the papers by Walker and by Garstang) and the assumption of luminosity proportional to the square root of the population as used by Berry (1976) and Pike (1976). Calculations by Garstang gave a theoretical relation which is in fair agreement with the observations by Berry. This, taken with the other successes of Garstang's model, seems to indicate that a linear relation between population and brightness is correct. It seems that the square root relationship is a device which makes substantial allowance for some of the defects of Berry's model, such as taking into account the extinction of the light coming from distant suburbs. Garstang examined whether there was any evidence to suggest that the luminosity-population departed from the simple $\mathrm{L} \propto \mathrm{P}$ law, and in particular whether there was evidence that the largest cities might be somewhat brighter than this law would predict. At first he thought that the evidence favored this (he suggested a law $\mathrm{L} \propto \mathrm{P}^{1.1}$ ) but later work did not seem to confirm this. Garstang's later work all assumes that $L \propto P$ in the absence of special circumstances. Garstang (1986) showed how his work could be used to calculate the limiting stellar magnitude visible to the naked eye, and he made (Garstang, 1985) a successful attempt to check the formulae of Weaver (1947) for very bright sky backgrounds. Calculations on Junipero Serra Peak were used to calibrate the natural sky background in the model.

One important conclusion which emerged from the calculations is the importance of improved shielding of lighting fixtures. Garstang (1986) estimated that at a distance of $40 \mathrm{~km}$ from Denver (population 1.3 million) a reduction by a factor 2 in the amount of light escaping above the horizontal would lead to a reduction of the zenith sky brightness from $21.20 \mathrm{mag} / \mathrm{sec}^{2}$ to $21.42 \mathrm{mag} / \mathrm{sec}^{2}$, and would increase the number of stars visible to the naked eye by $11 \%$.

Garstang $(1987,1988$ b) applied his model to the study of many observatories and prospective observatory sites. Included was a study of the sky brightness at many zenith distances and azimuths at Mount Graham. A few illustrative results are given in Table I. We tabulate the observed and calculated brightnesses and the quantity $\Delta \mathrm{m}$ which measures the increase in the calculated brightness over natural background. In general, we get good agreement with observations. In some cases the observed brightnesses are significantly brighter than brightnesses predicted from the standard model. The explanation seems to be that the natural sky background was brighter than our model assumed, most probably because of increased airglow intensity.

\section{PREDICTIONS OF SEASONAL EFFECTS}

The brightness of the night sky shows considerable variations of a short term nature. It must be asked whether there are regularities hidden in these apparently random fluctuations. Annual variations in the brightness seem not to have been detected observationally, but annual variations in the extinction have been observed. Recently published results include those of Lockwood and Thompson (1986), who summarized a long series of extinction measurements at the Lowell Observatory over the period May 1976 to April 1980 and showed the presence of an annual cycle. Rufener (1986) analyzed extensive data from the European Southern Observatory at La Silla. Extinction measurements in seven colors were made on 452 nights between November 1975 and March 1985, and 
the data were subjected to a very detailed analysis. The observations clearly showed the effect of the El Chichon volcano. An analysis of the observations obtained before the El Chichon eruption clearly showed an annual cycle, and a longer term trend was also apparent.

It is possible to use the extinction observations to make predictions of an annual cycle in the night sky brightness. Garstang (1986, equation (21)) gave a simple expression for the vertical extinction in the $\mathrm{V}$ band at an observatory in terms of the parameters of his model. Generalizing his expression to apply to any wavelength $\lambda$, we find that the extinction at wavelength $\lambda$ is

$$
\begin{aligned}
\mathrm{K}(\lambda) & =1.0857 \mathrm{~N}_{\mathrm{m}} \mathrm{r}_{\mathrm{R}} \exp (-\mathrm{cH}) \\
& \times\left\{5500 / \lambda^{4} \mathrm{c}^{-1} \exp (-\mathrm{cA})+(5500 / \lambda) \mathrm{CKa}^{-1} \exp (-\mathrm{aA})\right\}
\end{aligned}
$$

where $\mathrm{N}_{\mathrm{m}} \mathrm{r}_{\mathrm{R}}=0.0117 \mathrm{~km}^{-1}$ and $\mathrm{C}=11.778 . \mathrm{K}$ is the parameter which appears in the model to represent the aerosol content of the atmosphere. $c, a, H$ and $\mathrm{A}$ were defined earlier. $\mathrm{H}$ and $\mathrm{A}$ are measured in kilometers. ( $\mathrm{H}$ may often be taken as zero for observatories not too far from the coast, but a better estimate of the height should be used for inland observatories.) In Garstang's model, $\mathrm{c}=0.104 \mathrm{~km}^{-1}$ and $\mathrm{a}=0.657+0.059 \mathrm{~K} \mathrm{~km}^{-1}$. For the usual $\mathrm{B}$ band, $\lambda=$ $4400 \mathrm{~A}$ and for the usual $\mathrm{V}$ band $\lambda=5500 \mathrm{~A}$; appropriate values must be used for any other photometric system.

The data of Lockwood and Thompson (1986) can be analyzed by using the observed variations in the extinction to determine the corresponding variations of $K$. From the values of $K$ so obtained, a night sky brightness model can be used to calculate the variations of the night sky brightness due to the natural background and to the brightness caused by cities. We chose for a detailed calculation the night sky brightness at the Lowell Observatory Anderson Mesa station looking at a zenith distance of $45^{\circ}$ towards Flagstaff. The results are given in Table II. Variations of only $0.013 \mathrm{mag} / \mathrm{sec}^{2}$ are predicted in the B band natural sky background brightness, and $0.006 \mathrm{mag} / \mathrm{sec}^{2}$ in the $\mathrm{V}$ band. These are negligible for almost all purposes. Variations in the city contributions are predicted to be $0.034 \mathrm{mag} / \mathrm{sec}^{2}$ in the $\mathrm{B}$ band and $0.067 \mathrm{mag} / \mathrm{sec}^{2}$ in the $\mathrm{V}$ band. These might perhaps be detectable in an extensive observational program.

The data of Rufener (1986) can be analyzed in a similar manner, except that the extinction found at La Silla is slightly below the value predicted by equation (8) above with $\mathrm{K}=0$. To find a value of $\mathrm{K}$, a different approach was necessary. We chose to use the ratio of aerosol to molecule extinction given by Rufener, and to fit this ratio to the ratio of the two terms inside the braces in equation (8). We then used the resulting values of $\mathrm{K}$ to predict the night sky brightnesses. The results are given in Table II. The variations predicted are extremely small (a few thousandths of a mag/ $/ \mathrm{sec}^{2}$ ) and are unlikely to be detectable from observations.

There is another possibility for predicting seasonal changes, and that is from turbidity measurements, which depend on the measurements of the absorption of solar integrated radiation. The Linke turbidity factor $T$ is the ratio of the actual extinction for the integrated radiation to the Rayleigh extinction alone for an acrosol free dry atmosphere for the integrated radiation. A simple method of proceeding is to note that when dealing with the solar integrated radiation the molecular extinction involved is to a good approximation the mean value of the 
Rayleigh extinction coefficient weighted according to the spectral distribution of the solar radiation intensity. The aerosol extinction is the mean value of the aerosol extinction coefficient similarly weighted. Rough calculations showed that the mean wavelength for the molecular extinction could be taken as $5250 \mathrm{~A}$ and that for aerosol extinction as 6900A. Then in equation (8) we use $\lambda=$ $5250 \mathrm{~A}$ in the first term inside the braces and $\lambda=6900 \mathrm{~A}$ in the second term. The turbidity factor $\mathrm{T}$ was calculated as the ratio of the complete formula of equation (8) to the first term only. T has been measured at Tucson (Szymeber and Sellers, 1985) over a period of 27 years from 1956 to 1983 . We calculated the values of $\mathrm{K}$ corresponding to the mean values of $\mathrm{T}$, and then calculated the night sky brightnesses and the extinction coefficients for the zenith at Kitt Peak National Observatory. Results for the extreme months of the year are given in Table II. (Our results are slightly revised from the values given in Garstang, 1988a.) Variations of the extinction coefficients of up to 0.04 and variations of the manmade contributions to the night sky brightness of up to $0.03 \mathrm{mag} / \mathrm{sec}^{2}$ may be expected to arise from seasonal effects, variations in the night sky background from this cause are negligible.

The analysis of turbidity measurements as just outlined suffers from the major approximation that water vapor is neglected except as far as it can be regarded as included in the average aerosol. It is possible to improve on the method by correcting for water vapor if radiosonde determinations of precipitable water vapor content are also made. Calculations of the transmission of dust free atmospheres containing various amounts of water vapor were made by Yamamoto et al. (1968), who included Rayleigh scattering by oxygen and nitrogen and absorption by ozone, carbon dioxide and water vapor, and showed how to determine the aerosol optical depth from measurements of the actual solar radiation. The method was extended by Polavarapu (1978), who showed that it is useful to define the quantity $\Delta T=T-T$, where $T_{d f}$ is the Linke turbidity factor calculated for dust free air with the same water vapor content as the actual air. $\Delta T$ is the contribution to $T$ caused by aerosols alone. Szymber and Sellers (1985) applied this analysis to their measurements and gave values of $\Delta T$. We see from the results in Figure 2 of their paper that if we exclude the period when the $\mathrm{El}$ Chichon volcano was active $\mathrm{T}$ varies from about 0.2 to 0.6 during the year. This variation can be converted to a variation of $\mathrm{K}$ and night sky brightnesses calculated. The results for the two extreme months are given in Table I under the heading Kitt Peak (improved). There is an important qualitative difference between the two sets of results for Kitt Peak. It is that the values of $\mathrm{K}$ given by the improved calculation are smaller than the values given by the previous calculation. This is to be expected because the larger values of $\mathrm{K}$ include a contribution from the averaged water vapor absorption while the smaller values of $\mathrm{K}$ are after subtraction of the effects of water vapor. The smaller values are the more realistic, and they lead to somewhat lower values of the extinction and somewhat brighter man-made contributions to the night sky brightness, but the amplitudes of the seasonal changes are similar to those calculated previously.

There have been many other determinations of the turbidity coefficients, for example by Yamamoto et al. (1968), and annual cycles have been demonstrated at many sites. Our conclusion, however, remains that seasonal variations of night sky brightnesses are likely to be small and unimportant in comparison with random fluctuations from night to night and longer changes 
caused by the sunspot cycle.

\section{PREDICTIONS OF EFFECTS OF LONG TERM POPULATION GROWTH}

Another study which can be made is to determine the effect of future population growth on the sky brightness at observatories and sites. One must first obtain projections of population growth. Such projections are made by most states in the U.S.A., the particular agency responsible for projections varying from one state to another. Such projections are, of course, subject to substantial uncertainties because of unknown factors such as changes in economic conditions and population migration rates, which are in addition to more easily predicted factors such as fertility changes. One can simply scale the brightness at an observatory due to each polluting city with the predicted growth of that city and add the contributions to get the total brightness. A few examples of such predictions are given in Table III, and more extensive predictions are being made by Garstang (1989).

\section{CONCLUSIONS}

Considerable progress has been made since 1970 on modeling light pollution, and it is possible to interpret many of the observations which have been made, as well as to make useful predictions for sites which have not yet been studied in detail.

\section{ACKNOWLEDGMENTS}

I am indebted to D. Geisler and C. Pilachowski for communicating the results of their observations in advance of publication.

\section{REFERENCES}

Berry, R. L. 1976, J. Roy. Astron. Soc. Canada 70, 97-115.

Bertiau, F. C., de Graeve, E. and Treanor, P. J. 1973, Vatican Obs. Pub. 1, No. 4, 159-179.

Garstang, R. H. 1984, The Observatory 104, 196-197.

Garstang, R. H. 1985, J. Brit. Astron. Assoc. 95, 133.Garstang, R. H. 1986, Pub. Astron. Soc. Pacific 98, 364-375.

Garstang, R. H. 1987, in "The Identification, Optimization and Protection of Optical Observatory Sites" (eds. R. L. Millis, H. D. Ables and C. C. Dahn, Lowell Observatory, Flagstaff, AZ) pp. 199-202.

Garstang, R. H. 1988a, The Observatory 108, October issue.

Garstang, R. H. 1988b, Pub. Astron. Soc. Pacific (to be submitted.)

Garstang, R. H. 1989, Ann. Rev. Astron. Astrophys. (submitted).

Geisler, D. 1988, Nat. Opt. Astron. Obs. Newsletter No. 13, 22-23.

Geisler, D., Walker, A., Smith, D. and Weller, B. 1988, Nat. Opt. Astron. Obs. 
Newsletter No. $14,24$.

Lockwood, G. W. and Thompson, D. T. 1986, Astron. J. 92, 976-985.

Pike, R. 1976, J. Roy. Astron. Soc. Canada 70, 116-126.

Polavarapu, R. J. 1978, J. Appl. Met. 17, 1368-1374.

Rufener, F. 1986, Astron. Astrophys. 165, 275-286.

Smith, F. G. 1979 Trans. I.A.U. 17A, 220.

Szymber, R. J. and Sellers, D. 1985, J. Clim. Appl. Met. 24, 725-734.

Thomas, R. W. L., Modali, S. B. and Roose, R. G. 1973, Bull. Am. Astron Soc. 5, 391.

Treanor, P. J. 1973, The Observatory 93, 117-120.

Walker, M. F. 1970, Pub. Astron. Soc. Pacific 82, 672-698.

Walker, MM. F. 1973, Pub. Astron. Soc. Pacific 85, 508-519.

Walker, M. F. 1977, Pub. Astron. Soc. Pacific 89, 405-409.

Weaver, H. F. 1947, Pub. Astron. Soc. Pacific 59, 232-243.

Yamamoto, G., Tanaka, M. and Arao, K. 1968, J. Met. Soc. Japan 46, 287-299.

Yocke, M. A., Hogo, H. and Henderson, D. 1986, Pub. Astron. Soc. Pacific 98, 889-893. 
TABLE I Night sky brightness at observatories and sites

\begin{tabular}{|c|c|c|c|c|c|}
\hline & & & B & v & Notes \\
\hline \multirow[t]{6}{*}{ Kitt Peak } & Zenith & Calculated & 22.919 & 21.885 & 1 \\
\hline & & Observed & 22.909 & 21.922 & 2 \\
\hline & & $\Delta \mathrm{m}$ & 0.069 & 0.105 & 1 \\
\hline & ZD $45^{\circ}$ & Calculated & 22.728 & 21.644 & 1,3 \\
\hline & & Observed & 22.749 & 21.686 & 2,3 \\
\hline & & $\Delta \mathrm{m}$ & 0.106 & 0.147 & 1,3 \\
\hline \multirow[t]{3}{*}{ Mauna Kea } & Zenith & Calculated & 22.937 & 21.957 & 4 \\
\hline & & Observed & 23.05 & 21.97 & 5 \\
\hline & & $\Delta \mathrm{m}$ & 0.022 & 0.023 & 4 \\
\hline \multirow[t]{2}{*}{ MeDonald } & Zenith & Calculated & 22.986 & 21.984 & 4 \\
\hline & & $\Delta \mathrm{m}$ & 0.007 & 0.009 & 4 \\
\hline \multirow{5}{*}{\multicolumn{2}{|c|}{ Cerro Tololo Zenith }} & Calculated & 22.975 & 21.979 & 6 \\
\hline & & Observed & 22.91 & 21.99 & 7 \\
\hline & & $\Delta \mathrm{m}$ & 0.008 & 0.009 & 6 \\
\hline & & Calculated & 22.56 & 21.57 & 8 \\
\hline & & Observed & 22.54 & 21.58 & 9 \\
\hline
\end{tabular}

1. Standard model of Garstang (1986, 1988b), brightness interpolated for 1987 using calculations for 1980 and 1990.

2. Average values for 1987 , communicateed to me by C. Pilachowski.

3. Averaged over all azimuths.

4. Standard model, 1980 populations

5. D. Tholen, 1987, communicated to me by C. Pilachowski.

6. Used light emission per head one tenth of standard model.

7. Geisler (1988).

8. Airglow contribution to sky background increased by $75 \%$.

9. Geisler et al. (1988). 
TABLE II Predicted variations* in the night sky brightness

\begin{tabular}{rcccr} 
Extinction & Natural background & \multicolumn{2}{r}{ Cities } \\
B $V$ & $B$ & $V$ & $\Delta m$ & $\Delta m$ \\
& & & $B$ & V
\end{tabular}

Lowell Observatory

$\begin{array}{llllllll}\text { May } & 0.38 & 0.32 & 0.17 & 22.843 & 21.795 & 0.429 & 0.433 \\ \text { December } & 0.17 & 0.28 & 0.13 & 22.830 & 21.789 & 0.395 & 0.364 \\ & & & & & & & \\ \begin{array}{l}\text { E.S.0. } \\ \text { February } \\ \text { August }\end{array} & 0.59 & 0.26 & 0.12 & 22.981 & 21.987 & 0.001 & 0.000 \\ & 0.10 & 0.24 & 0.10 & 22.977 & 21.985 & 0.001 & 0.001 \\ \text { Kitt Peak } & & & & & & & \\ \text { July } & 1.30 & 0.35 & 0.19 & 22.999 & 21.996 & 0.022 & 0.034 \\ \text { December } & 0.75 & 0.31 & 0.16 & 22.992 & 21.996 & 0.046 & 0.067 \\ & & & & & & & \\ \text { Kitt Peak } & (\text { improved) } & & & & & \\ \text { July } & 0.47 & 0.30 & 0.15 & 22.989 & 21.991 & 0.057 & 0.076 \\ \text { December } & 0.15 & 0.27 & 0.12 & 22.984 & 21.988 & 0.069 & 0.100\end{array}$

* More extensive tables of data for the Lowell Observatory and for Kitt Peak are given in Garstang (1988a). The above table shows only the predictions for the two extreme months.

TABLE III Prediction of future night sky brightness increases*

\begin{tabular}{|c|c|c|c|c|c|}
\hline Date & $\begin{array}{l}\text { Kitt } \\
\text { Peak }\end{array}$ & MeDonald & $\begin{array}{c}\text { Mt. } \\
\text { Graham }\end{array}$ & $\begin{array}{c}\text { Sacramento } \\
\text { Peak }\end{array}$ & $\begin{array}{c}\text { Mt. } \\
\text { Hopkin }\end{array}$ \\
\hline 1980 & 0.085 & 0.008 & 0.044 & 0.11 & 0.14 \\
\hline 1990 & 0.114 & 0.010 & 0.054 & 0.14 & 0.18 \\
\hline 1995 & 0.130 & 0.010 & 0.059 & 0.16 & 0.21 \\
\hline 2000 & 0.147 & 0.011 & 0.064 & 0.17 & 0.24 \\
\hline 2005 & 0.165 & 0.012 & 0.069 & 0.19 & 0.28 \\
\hline
\end{tabular}

*All quantities quoted are the magnitude increases $\Delta \mathrm{m}$ caused by man-made light pollution for $V$ magnitudes at the zenith. 